\title{
Holonomic Systems of Linear Differential Equations and Feynman Integrals
}

\author{
by \\ Masaki KASHIWARA* and Takahiro KAWAI**
}

The purpose of this report is to show that the (generalized) Feynman integral should satisfy a holonomic system***) of linear differential equations. We also discuss the analyticity of the defining function ${ }^{\dagger)}$ of the Feynman amplitude in complex domain as a corollary of this result. Our main result, i.e. the existence of holonomic system, gives an affirmative answer to the conjecture of Regge [15], who first understood and emphasized the importance of the role of systems of differential equations in the investigation of Feynman integrals. In his report a homological approach to this problem is suggested. It is very illuminating but seems to be accompanied with many technical difficulties, as Professor Regge himself points out in the report. This important property of the Feynman integral has also been conjectured and proved in simple cases by Sato [16] independently and in a little different context See also BarucchiPonzano [1], Kawai-Stapp [11], [12] and references cited there. Note that Kawai-Stapp ([11], [12]) discusses the $S$-matrix itself, not the individual Feynman integral, as Sato [16] originally proposed. We also give the diagramatic description of the characteristic variety of the system involved. It enjoys a nice physical interpretation as is shown by Kashi-

Received August 28, 1976.

* Mathematical Institute, Nagoya University and Department of Mathematics, Harvard University.

Supported by the National Science Foundation.

** Research Institute for Mathematical Sciences, Kyoto University and Department of Mathematics, University of California, Berkeley.

Supported by Miller Institute for Basic Research in Science.

*** It is called "maximally overdetermined system" in Sato-Kawai-Kashiwara [17], Kashiwawa-Kawai [7] and Kawai-Stapp [11].

${ }^{+}$A defining function of a hyperfunction is by definition a holomorphic function whose boundary value attains the hyperfunction in question. See Theorem 4 below for the precise definition. 
wara-Kawai-Stapp [10]. We discuss the case of the generalized Feynman integral after Speer [19], though we do not make full use of the parametric representation of the integral except in the proof of Theorem 7 .

In this report all relevalent particles are supposed to be massive and spinless. In the sequel we use the same notions and notations as in Kawai-Stapp [11], [12], Nakanishi [14], Sato-Kawai-Kashiwara [17] and Speer [19] and we do not repeat their definitions here.

Since the main purpose of this report is to discuss every Feynman integral of any order, we discuss in a separate paper (Kashiwara-KawaiOshima [9]) more specific topics which should be covered also under the same title of this report. There, for a limited class of Feynman integrals we discuss the explicit form of Feynman integrals near the Landau surface (of the first kind and the second kind) and the hierarchical principle (Landshoff-Olive-Polkinghorne [13]) in terms of holonomic system of (micro-) differential equations.

We would like to express our heartiest thanks to Professor Regge and Professor Stapp for many stimulating and interesting discussions with them.

The detailed argument of this report will be given in a forthcoming book of Kashiwara-Kawai [8].

Our argument essentially relies on the following theorem. (Cf. Kashiwara-Kawai [7], Kashiwara [5], Kashiwara-Kawai-Stapp [10].)

Theorem 1. Let $\varphi_{j}(x)(j=1, \cdots, d)$ and $f_{l}(x) \quad(l=1, \cdots, N)$ be real valued real analytic functions defined on a real analytic manifold $M$. Denote by $X$ a complexification of $M$. Denote by $Y$ the variety defined by $\left\{x \in X ; \varphi_{1}(x)=\cdots=\varphi_{d}(x)=0\right\}$. Assume that $Y$ has codimension $d$ in $X$ and that $Y$ is irreducible and nonsingular except for proper analytic subset $Y_{\text {sing }}$ of $Y$. Assume that $\left.f_{l}\right|_{Y} \neq 0(l=1, \cdots, N)$ and that $Y_{\text {sing }}$ is contained in $\left\{x \in X ; \prod_{l=1}^{N} f_{l}(x)=0\right\}$. Assume that $\Phi(x)=$ $=\prod_{j=1}^{d} \delta\left(\varphi_{j}(x)\right) \prod_{i=1}^{N}\left(f_{l}(x)+i 0\right)^{\lambda_{l}}$ is a well-defined hyperfunction in $x \in M$ which depends holomorphically on $\lambda=\left(\lambda_{1}, \cdots, \lambda_{N}\right)$ in a neighborhood of 
$\lambda^{0}=\left(\lambda_{1}{ }^{0}, \cdots, \lambda_{N}{ }^{0}\right){ }^{*} \quad$ Then $\Phi(x)$ satisfies a holonomic system $\mathscr{M}=\mathscr{M}_{2}$ of linear differential equations. Further the characteristic variety of $\mathcal{M}$ is contained in $W_{0}^{\prime}=\left\{(x, \eta) \in T^{*} X\right.$; there exist a sequence $x_{m} \in X$ such that $\varphi_{j}\left(x_{m}\right)=0(j=1, \cdots, d)$ and that converges to $x$ and sequences $s_{m}=\left(s_{1}{ }^{(m)}, \cdots, s_{N}{ }^{(m)}\right) \in \mathbb{C}^{N}$ and $t_{m}=\left(t_{1}{ }^{(m)}, \cdots, t_{d}{ }^{(m)}\right) \in \mathbb{C}^{d}$ such that $s_{l}{ }^{(m)} f_{l}\left(x_{m}\right)$ converges to zero $(l=1, \cdots, N)$ and that $\sum_{l=1}^{N} s_{l}{ }^{(m)} \operatorname{grad}_{x} f_{l}\left(x_{m}\right)+\sum_{j=1}^{d} t_{j}^{(m)} \times$ $\times \operatorname{grad}_{x} \varphi_{j}\left(x_{m}\right)$ converges to $\left.\eta\right\}$.

As discussed in Kashiwara-Kawai [7], the proof of this theorem is essentially based on the desigularization theorem of Hironaka (HironakaLejeune-Teissier [3]).

The generalized Feynman integral $F_{D}(p ; \lambda)$ associated with a Feynman diagram $D$ with $n$ vertices, $n^{\prime}$ external lines and $N$ internal lines is by definition the following integral up to a constant factor. (Cf. Nakanishi [14], Speer [19] and the references cited there.)

$$
\int \frac{\prod_{j=1}^{n} \delta^{4}\left(\sum_{r=1}^{n^{\prime}}[j: r] p_{r}+\sum_{l=1}^{N}[j: l] k_{l}\right)}{\prod_{l=1}^{N}\left(k_{l}{ }^{2}-m_{l}{ }^{2}+i 0\right)^{\lambda_{l}}} \prod_{l=1}^{N} d^{4} k_{l} .
$$

Clearly it can be rewritten in the form

$$
\delta^{4}\left(\sum_{j, r}[j: r] p_{r}\right) \int \frac{\prod_{j=1}^{n-1} \delta^{4}\left(\sum_{r=1}^{n^{\prime}}[j: r] p_{r}+\sum_{l=1}^{N}[j: l] k_{l}\right)}{\prod_{l=1}^{N}\left(k_{l}{ }^{2}-m_{l}{ }^{2}+i 0\right)^{\lambda_{l}}} \prod_{l=1}^{N} d^{4} k_{l} .
$$

The function obtained by factorizing out the over-all $\delta$-function $\delta^{4}\left(\sum_{j, r}[j: r] p_{r}\right)$ from the generalized Feynman integral, i.e. the second factor in $\left(1^{\prime}\right)$, is called by definition the generalized Feynman amplitude.

Since the integral given by (1) is an improper integral, we investigate a corresponding integral defined on a suitable compactification of $\boldsymbol{R}^{4 N}$. Here we use $\left[P\left(\mathbb{R}^{4}\right)\right]^{N}$ as a preferred compactification of $\mathbb{R}^{4 N}$ $\cong\left(\mathbb{R}^{4}\right)^{N}$, that is, we use the projective compactification with respect to each internal momentum $k_{l} \in \boldsymbol{R}^{4}$. This compactification procedure is known

* By making use of the desingularization theorem of Hironaka, it is easy to see that $\Phi(x)$ is well-defined and holomorphic in $\lambda$ for $\operatorname{Re} \lambda_{l} \gg 0(l=1, \cdots, N)$. 
to give rise to the same function as that discussed by Speer [19] in terms of parametric representations. (See Sato-Miwa-Oshima-Jimbo [18].)

Using this compactification of $\boldsymbol{R}^{4 N}$, we investigate the integral whose integrand $\Phi_{D}$ is expressed in the following form (2) near the points at infinity by making use of the homogeneous coordinate $\left(K_{l}, c_{l}\right)_{1 \leqq l \leq N}$ on $\left(P\left(\boldsymbol{R}^{4}\right)\right)^{N}$.

$$
\begin{aligned}
& \Phi_{D}(p, K, c ; \lambda) \omega(K, c)= \\
& =\frac{\prod_{j=1}^{n} \delta^{4}\left(\sum_{r=1}^{n^{\prime}}[j: r] \prod_{\left\{l^{\prime} ;\left[j ; l^{\prime}\right] \neq 0\right\}} c_{l^{\prime}} p_{r}+\sum_{l=1}^{N}[j: l] \frac{\prod_{\left.l^{\prime} ;\left[j: l^{\prime}\right] \pm 0\right\}} c_{l^{\prime}}}{c_{l}} K_{l}\right)}{\prod_{l=1}^{N}\left(K_{l}{ }^{2}-c_{l}{ }^{2} m_{l}{ }^{2}+i 0\right)^{\lambda_{l}}} \times \\
& \quad \times \prod_{l=1}^{N}\left|c_{l}\right|^{\mu_{l}} \omega\left(K_{1}, c_{1}\right) \cdots \omega\left(K_{N}, c_{N}\right) .
\end{aligned}
$$

Here $\mu_{l} \equiv 2 \lambda_{l}+4 \#\{j ;[j: l] \neq 0\}-5=2 \lambda_{l}+3$ and $\omega\left(K_{l}, c_{l}\right)$ is the volume element on $P\left(\boldsymbol{R}^{4}\right)$.

It is known (Sato-Miwa-Oshima-Jimbo [18]) that $\Phi_{D}(p, K, c ; \lambda) \omega(K, c)$ is a well-defined differential form with hyperfunction coefficients for generic $\lambda_{l}$ if $m_{l} \neq 0$, that is, $\Phi_{D}(p, K, c ; \lambda) \omega(K, c)$ depends meromorphically on $\lambda . *)$

Since $\Phi_{D}$ satisfies conditions of Theorem $1,{ }^{* *}$ we conclude that $\Phi_{D}$ satisfies a holonomic system of linear differential equations. Then Lemma 5 of Kashiwara-Kawai [7] proves that $F_{D}(p ; \lambda)=\int \Phi_{D} \omega$ should satisfy a holonomic system $\mathscr{M}=\mathscr{M}_{\lambda}$ of linear differential equations in $p$ as long as $\lambda$ is generic. Furthermore, combining Lemma 1 of this note and (7) of Kashiwara-Kawai [7], we can find the maximum possible set for the characteristic variety of $\mathcal{M}$. Thus we have the following theorem:

Theorem 2. Generalized Feynman integral $F_{D}(p ; \lambda)$ defined by $\int \Phi_{D} \omega$ satisfies a holonomic system $\mathscr{M}=\mathscr{M}(D, \lambda)$ of linear differential equations in $p$ as long as $\lambda=\left(\lambda_{1}, \cdots, \lambda_{N}\right)$ is generic. The characteristic variety of $\mathscr{M}$ is contained by the following set $\widetilde{\mathcal{L}}(D)$ (extended Lan-

* Though the well-definedness of $\boldsymbol{\emptyset}_{D}$ is discussed only for external diagram $D$ in SatoMiwa-Oshima-Jimbo [18], their argument applies to the general case.

** Note that $|f|^{\lambda}$ is a linear combination of $(f+i 0)^{\lambda}$ and $(f-i 0)^{\lambda}$ as long as $\lambda$ is not an integer. 
dau variety associated with Feynman diagram $D)$. Here $X$ denotes $\mathbb{C}^{4 n^{\prime}}$.

$$
\widetilde{L}(D)=\left\{(p ; u) \in T^{*} X ; \text { there exists a sequence of scalars } c_{l}{ }^{(m)}\right. \text { and }
$$
$\alpha_{l}{ }^{(m)}(l=1, \cdots, N)$ and four-vectors $p_{r}{ }^{(m)}, u_{r}{ }^{(m)}\left(r=1, \cdots, n^{\prime}\right), k_{l}{ }^{(m)}(l=1$, $\cdots, N)$ and $v_{j}^{(m)}(j=1, \cdots, n)$ which satisfy the following relation (3)\}.

$$
\begin{cases}p_{r}{ }^{(m)} \longrightarrow p_{r} & \left(r=1, \cdots, n^{\prime}\right) \\ u_{r}{ }^{(m)} \longrightarrow u_{r} & \left(r=1, \cdots, n^{\prime}\right) \\ \sum_{r=1}^{n^{\prime}}[j: r] p_{r}{ }^{(m)}+\sum_{l=1}^{N}[j: l] k_{l}{ }^{(m)}=0 & (j=1, \cdots, n) \\ u_{r}{ }^{(m)}=\sum_{j=1}^{n}[j: r] v_{j}{ }^{(m)} & \left(r=1, \cdots, n^{\prime}\right) \\ \sum_{j=1}^{n}[j: l] v_{j}{ }^{(m)}+\alpha_{l}{ }^{(m)} k_{l}{ }^{(m)} & \\ c_{l}{ }^{(m)} & (l=1, \cdots, N) \\ \alpha_{l}{ }^{(m)}\left(k_{l}{ }^{(m) 2}-m_{l}{ }^{2}\right) \longrightarrow 0 & (l=1, \cdots, N) \\ c_{l}{ }^{(m)} \text { is bounded } & (l=1, \cdots, N) \\ c_{l}{ }^{(m)} k_{l}{ }^{(m)} \text { is bounded } & (l=1, \cdots, N) \\ {\left(c_{l}{ }^{(m)}, c_{l}{ }^{(m)} k_{l}{ }^{(m)}\right) \longrightarrow 0}_{l}^{\longrightarrow} & (l=1, \cdots, N)\end{cases}
$$

Remark 1. Note that the usual Landau variety is not necessarily holonomic, ${ }^{*}$ while the extended Landau variety is always holonomic. This indicates that the employment of the extended Landau varieties is more natural than the employment of the usual Landau varieties in our context, i.e. from the view point of holonomic systems, which are expected to control the behavior of the Feynman integral at the points far away from the physical region. (Cf. Theorem 5 below.) In fact, it is obvious that the extended Landau variety reduces to the usual Landau variety in the positive- $\alpha$ region (i.e., under the additional assumption of reality of every quantity involved, positivity of $\alpha_{l}$ 's and finiteness of $k_{l}$ 's), while there is no a priori reason for believing that (the complexification of) the usual Landau variety (even after being supplemented by the second type sin-

* In this sense the terminology "Landau holonomic variety" used by Sato-MiwaOshima-Jimbo [18] is an abuse of the language and seems to be misleading. 
gularities) should give the maximum possible set of the singularity for Feynman integrals outside positive- $\alpha$ region. One should recall that the celebrated argument of Landau and Nakanishi for the derivation of Landau equations (or Landau-Nakanishi equations) is essentially based on the positivity of $\alpha$.

Remark 2. Theorem 2 holds without any change even when some internal masses $m_{l}$ 's are zero.

Since S.S. $F_{D}(p ; \lambda)$, hence S.S. $f_{D}(p ; \lambda)$, is confined to the positive- $\alpha$ Landau variety (Chandler[2], Sato-Miwa-Oshima-Jimbo [18]), it is easy to see that there exists a unique holomorphic function $\tilde{f}_{D}(p ; \lambda)$ in $p \in \mathbb{C}^{4 n^{\prime}}$ with $\sum_{j, r}[j: r] p_{r}=0$ whose boundary value attains generalized Feynman amplitude $f_{D}(p ; \lambda)$. In fact, in view of Proposition 1.5.4. of Sato-KawaiKashiwara [17] Chapter I, it suffices to show that S.S. $f_{D}(p ; \lambda)$ is contained in a proper convex set in the sense of the proposition quoted above. This fact immediately follows from Landau equations as follows:

$$
\begin{aligned}
& \sum_{r=1}^{n^{\prime}}\left\langle p_{r}, u_{r}\right\rangle \\
= & \sum_{r=1}^{n^{\prime}}\left\langle p_{r}, \sum_{j=1}^{n}[j: r] v_{j}\right\rangle \\
= & \sum_{r, j}[j: r]\left\langle p_{r}, v_{j}\right\rangle \\
= & -\sum_{j}\left\langle\sum_{l}[j: l] k_{l}, v_{j}\right\rangle \\
= & -\sum_{j, l}[j: l]\left\langle k_{l}, v_{j}\right\rangle \\
= & -\sum_{l} \alpha_{l} k_{l}{ }^{2}=-\sum_{l} \alpha_{l} m_{l}{ }^{2}\langle 0 .
\end{aligned}
$$

Since $f_{D}(p ; \lambda)$ satisfies holonomic system $\mathscr{M}$ of linear differential equations, $\tilde{f}_{D}(p ; \lambda)$ should satisfy the same system $\mathscr{M}$. Obviously this fact must give a strong restriction on the functional character of $\tilde{f}_{D}(p ; \lambda)$. The first general result in this direction is following Theorem 3. Some applications of this result to the rigorous derivation of pole-factorization theorem will be found in Iagolnitzer-Stapp [14]. 
Theorem 3. Let $\mathscr{M}$ be a holonomic system of micro-differential equations. Assume that the characteristic variety $V$ of $\mathcal{M}$ is the conormal bundle of a hypersurface $\left\{x ; x_{1}=0\right\}$, i.e. $\left\{(x, \eta) ; x_{1}=0, \eta_{2}=\cdots\right.$ $\left.=\eta_{n}=0\right\}$. Let $f(x)$ be a microfunction solution of $\mathcal{M}$ defined in a neighborfood of $\left\{(x, \sqrt{-1} \eta) ; x_{1}=0, \eta_{1}>0, \eta_{2}=\cdots=\eta_{n}=0\right\}$. Then $f(x)$ has the following form:

$$
\sum_{l, \alpha, j} P_{l}\left(x, D_{x}\right)\left(x_{1}+i 0\right)^{\alpha}\left(\log \left(x_{1}+i 0\right)\right)^{j} .
$$

Here indices $l, \alpha$ and $j$ run over a finite set, $j$ 's are integers and $P_{l}\left(x, D_{x}\right)$ is a micro-differential operator of possibly infinite order.

The proof of this theorem is given by reducing to the problem of the 1-dimensional case by making use of Theorem 5.3.1 of Sato-KawaiKashiwara [17] Chapter II.

When the characteristic variety of $\mathcal{M}$ is more complicated than the case treated by Theorem 3, we cannot expect to have such a concrete expression of a microfunction solution $f(x)$ of $\mathscr{M}$. However, we still have the following general result when $\mathscr{M}$ is a holonomic system of linear differential equations. In case $\mathcal{M}$ is a holonomic system of microdifferential equations, the argument becomes very complicated, so we will discuss it in a separate paper. Here we only mention that the results of Kashiwara-Kawai [6] turn out to be useful in this case.

Theorem 4. Let $M$ be a real analytic manifold and $X$ its complexification. Let $\mathscr{M}$ be a holonomic system of linear differential equations defined on a complex manifold $X$. Let $V \subset P^{*} X$ be the characteristic variety of $\mathscr{M}$. Denote by $\pi$ the projection from $P^{*} X$ to $X$. Let $Y$ be the union of the components of $\pi(V)$ of codimension 1 . Let $f(x)$ be a hyperfunction solution of $\mathscr{M}$ defined on $M$. Assume that there exists $\varphi \in \Gamma(U, \widetilde{\AA})$ for an open subset $U$ of $\sqrt{-1} S M$ with convex fiber such that $\left.f=\alpha(\varphi){ }^{*}\right)$ Then we can find a multivalued analytic continuation $\tilde{\varphi}$ of $\varphi$ which is analytic outside $Y$.

* See $§ 1.5$ of Sato-Kawai-Kashiwara [17] Chapter I for the definition of sheaf $\widetilde{\mathcal{A}}$ and map $\alpha$. 
This result applied to the generalized Feynman amplitude entails the following:

Theorem 5. Denote by $Y$ the linear subspace $C^{4 n^{\prime}-4}$ of $X=C^{4 n^{\prime}}$ defined by $\sum_{j, r}[j: r] p_{r}=0$. Define the extended Landau "surface" $\widetilde{L}(D)$ associated with a Feynman diagram $D$ by the projection of the extended Landau variety $\widetilde{\mathcal{L}}(D) \subset P^{*} Y$ to the base manifold $\left.Y{ }^{*}\right)$ Denote by $\widetilde{L}_{0}(D)$ the union of the components of $\widetilde{L}(D)$ of codimension 1. Then the defining function $\tilde{f}_{D}(p ; \lambda)$ of generalized Feynman amplitude $f_{D}(p ; \lambda)$ can be extended as a multivalued analytic function defined and analytic on $Y-\widetilde{L}_{0}(D)$.

Remark. This result is a basic one in understanding the monodromy problem for Feynman amplitudes from the view point of holonomic systems. This topic will be discussed elsewhere. Here we only note that the existence of holonomic system entails that all the branches of analytically continued function are locally a linear combination of finitely many fixed branches. This is a starting point of the investigation of the monodromy groups relative to Feynman integrals. (Cf. Regge [15] and references cited there.)

Thus far, we have discussed generalized Feynman integrals keeping parameters $\lambda_{l}$ 's to be generic. However, it is also possible to discuss the renormalized integrals by making use of the celebrated procedure of analytic renormalization due to Speer [19].

In order to find the holonomic system which the renormalized integral should satisfy, we apply the following general result:

Theorem 6. Let $f(x ; \lambda)$ be a hyperfunction in $x$ which depends

* Though extended Landau variety $\tilde{\mathcal{L}}(\mathrm{D})$ was defined to be a subvariety of $T^{*} X$ (Theorem 2), the same equations define a variety in $T^{*} Y$ under the convention that $(p ; u)$ and $\left(p^{\prime} ; u^{\prime}\right)$ define the same point if $p=p^{\prime}$ and if $u_{r}=u_{r}+a\left(r=1, \cdots, n^{\prime}\right)$ for a fourvector $a$. Furthermore, here we have excluded the zero-section of $\widetilde{\mathcal{L}}(D)$, i.e. the component where $u=0$, from the investigation so that we may consider it as a subvariety of $P^{*} X$. Note that Feynman amplitude $f_{D}(p ; \lambda)$ is well-defined only on $Y$. 
maromorphically on $\lambda=\left(\lambda_{1}, \cdots, \lambda_{N}\right)$. Assume that there exist linear differential operators $P_{j}\left(x, D_{x} ; \lambda\right)(j=1, \cdots, N)$ in $\mathscr{D}_{x} \otimes C\left[\lambda_{1}, \cdots, \lambda_{N}\right]$ such that $P_{j}\left(x, D_{x} ; \lambda\right) f(x ; \lambda)=0(j=1, \cdots, N)$ holds. Assume further that $P_{j}\left(x, D_{x} ; \lambda_{0}\right) f(x)=0(j=1, \cdots, N)$ defines a holonomic system for any fixed $\lambda=\lambda_{0}$. Assume further that $\prod_{K \in\{1, \ldots, N\}}\left(\sum_{i \in K}\left(\lambda_{l}-1\right)\right) f(x ; \lambda)$ defines a hyperfunction in $x$ which depends holomorphically on $\lambda$ in a neighborhood of $\lambda_{l}=1 \quad(l=1, \cdots, N)$. Then

$$
\mathscr{W}_{N}(f)=\frac{1}{N !} \sum_{\sigma \in \mathbb{\Phi}(N)} \frac{1}{(2 \pi i)^{N}} \oint_{\sigma_{\sigma(1)}} d \lambda_{1} \cdots \oint_{C_{\sigma(N)}} d \lambda_{N} \frac{f(x ; \lambda)}{\left(\lambda_{1}-1\right) \cdots\left(\lambda_{N}-1\right)}
$$

is a well-defined hyperfunction which satisfies a holonomic system of linear differential equations in $x$, if we choose the contours $C_{k}$ by $\left\{\lambda \in \mathbb{C} ;|\lambda-1|=R_{k}\right\}$ for constants $R_{k}$ satisfying

$$
\begin{aligned}
& 0<R_{1}<\cdots<R_{N} \ll 1 \\
& R_{k}>\sum_{j=1}^{k-1} R_{j} .
\end{aligned}
$$

Here $\sigma$ denotes a permutation on $\{1, \cdots, N\}$.

By making use of parametric representation of generalized Feynman amplitude $f_{D}(p ; \lambda)$, Speer [19] has shown that it is holomorphic in $\lambda$ after being multiplied by a factor $\prod_{K \subset\{1, \ldots, N\}}\left(\sum_{i \in K}\left(\lambda_{l}-1\right)\right)$ and that $\mathscr{W}_{N}\left(f_{D}(p ; \lambda)\right)$ gives rise to a renormalized amplitude. Therefore, by virtue of Theorem 2 and Theorem 6 one obtains following Theorem 7 .

Theorem 7. Analytically renormalized Feynman amplitude $W_{N}\left(f_{D}(p ; \lambda)\right)$ satisfies a holonomic system of linear differential equations whose characteristic variety is contained in the extended Landau variety $\widetilde{\mathcal{L}}(D)$.

\section{References}

[1] Barucchi, G. and G. Ponzario, Differential equations for one-loop generalized Feynman integrals, J. Math. Phys. 14 (1973), 390-401.

[2] Chandler, C., Some physical region mass shell properties of renormalized Feynman integrals, Commun. math. Phys., 19 (1970), 169-188.

[3] Hironaka, H., M. Lejeune, and B. Teissier, Résolution des singularités des espace 
analytiques complexes. To appear.

[4] Iagolnitzer, D. and H. P. Stapp, Pole factorization theorem in $S$-matrix theory. To appear.

[5] Kashiwara, M., On the rationality of roots of $b$-functions. To appear.

[6] Kashiwara, M. and T. Kawai, Micro-hyperbolic pseudo-differential operators I, $J$. Math. Soc. Japan, 27 (1975), 359-404.

[7] - Micro-local properties of $\prod_{j=1}^{n} f_{j \neq}^{s_{j}}$, Proc. Japan Acad., 51 (1975), 270-272.

[8] - Theory of micro-differential equations, Kōdansha, Tokyo. In preparation.

[9] Kashiwara, M., T. Kawai and T. Oshima, Micro-differential equation theoretic approach to Feynman integrals. In preparation.

[10] Kashiwara, M., T. Kawai and H. P. Stapp, Micro-analytic structure of the $S$-matrix and related functions. To appear. A summary is given in this proceeding.

[11] Kawai, T. and H. P. Stapp, Micro-local study of the $S$-matrix singularity structure, Lecture Notes in Physics, No. 39, Springer, Berlin-Heidelberg-New York, pp. 38-48 (1975).

[12] - Discontinuity formula and Sato's conjecture. This proceeding.

[13] Landshoff, P. V., D. I. Olive and J. C. Polkinghorne, The hierarchical principle in perturbation theory, Nuovo Cimento 43 (1966), 444-453.

[14] Nakanishi, N., Graph Theory and Feynman Integrals. Gordon and Breach, New York-London-Paris (1971).

[15] Regge, T., Algebraic topology methods in the theory of Feynman relativistic amplitudes, Report of Battelle Rencontres, Benjamin, New York, pp. 433-458 (1968).

[16] Sato, M., Recent development in hyperfunction theory and its application to physics, Lecture Notes in Physics. No. 39, Springer, Berlin-Heidelberg-New York, pp. 13-29 (1975).

[17] —, T. Kawai and M. Kashiwara, Microfunctions and pseudo-differential equations, Lecture Notes in Math. No. 287, Springer, Berlin-Heidelberg-New York, pp. 265-529 (1973).

[18] Sato, M., T. Miwa, M. Jimbo and T. Oshima, This proceeding.

[19] Speer, E. R., Generalized Feynman Amplitudes, Annals of Mathematics Studies, No. 62, Princeton University Press, Princeton (1969). 